\title{
A DEEP LARGE AREA SEARCH FOR LOW LUMINOSITY STARS
}

\author{
HUGH R.A. JONES \\ Institute of Astronomy, University of Tokyo, 2-21-1 Osawa, Tokyo 181 \\ Astrophysics Group, Liverpool JM University, Byrom Street, Liverpool L3 $3 A F$ \\ AND \\ MIKE R. S. HAWKINS \\ Royal Observatory Edinburgh, Blackford Hill, Edinburgh EHg зHJ
}

In a recent survey for faint red stars from a digital stack of Schmidt plates a number of candidate objects were identified. Parallax's for three of these objects have been reported showing them to have luminosities which interpreted within the available evolutionary models indicate them to be good brown dwarf candidates. Here we examine spectra of these objects and others from the plate stack. Using standard spectral indices we find that for a given spectral type their spectra are more consistent with the Pleiades brown dwarfs (PPL 15, Teide 1 and Calar 3) than with standard late-type $\mathrm{M}$ dwarfs. Our interpretation is that this is due to their selection by $R_{F}-I_{N}$ colours which at values $>3$ preferentially selects objects with relatively low gravities. For late-type $M$ dwarfs and brown dwarfs low gravities are expected to be a reliable indication of youth. We also notice that the stack objects generally have strong $\mathrm{FeH}$ absorption for their spectral type. Current model atmospheres suggest that $\mathrm{FeH}$ strongly increases in strength toward lower metallicities and lower temperatures. We believe that this is not consistent with the available observational evidence from late-type $\mathrm{M}$ dwarfs. It is possible that solid $\mathrm{Fe}$ is forming in the low temperature atmospheres relatively depleting $\mathrm{FeH}$ strengths toward lower temperatures. We find some evidence that for dwarfs at low temperatures dust formation is less prevalent in lower gravity objects suggesting that dwarfs at low temperatures stronger $\mathrm{FeH}$ may be an indication of youth. In addition to the spectral evidence the three stack objects whose parallax's have been measured show small tangential velocities which is a further indication of youth.

We have constructed preliminary models for the number of brown dwarfs and $\mathrm{M}$ dwarfs expected from the digital stack survey. We find that most of the objects are brown dwarfs less than $1 \mathrm{Gyr}$ old and because of this we make models using a time-dependent scale height. $\mathrm{M}$ dwarfs will dominate over brown dwarfs until around $I=18$ for $\alpha=-2.35$ and $I=20$ for $\alpha=0.00$. For the case of $\alpha=0.00$ the survey would be expected to detect few brown dwarfs. Noting the large uncertainties in constructing reliable models we believe our findings are more consistent with $\alpha=-2.35$. Overall their low luminosities, peculiar spectra, low tangential velocities and the expectation of detecting young brown dwarfs suggest that the stack objects are young brown dwarfs some of which may have very low masses. The main problem with such an interpretation is that their position in the HR diagram does not easily fit with the Pleiades sequence of brown dwarfs or the predictions of evolutionary models. However for late-type $\mathrm{M}$ dwarfs there is evidence that evolutionary models and synthetic spectra do yet give consistent results. The clear resolution of these issues probably requires further parallax measurements and follow-up observations of the new objects together with considerable work on high-temperature dust opacities and their inclusion in evolutionary models. 